University of Nebraska - Lincoln

DigitalCommons@University of Nebraska - Lincoln

Peter Dowben Publications

Research Papers in Physics and Astronomy

July 1989

\title{
Magnetic ordering of thin Gd overlayers
}

D. LaGraffe

Syracuse University

Peter A. Dowben

University of Nebraska-Lincoln, pdowben@unl.edu

M. Onellion

University of Wisconsin-Madison

Follow this and additional works at: https://digitalcommons.unl.edu/physicsdowben

Part of the Physics Commons

LaGraffe, D.; Dowben, Peter A.; and Onellion, M., "Magnetic ordering of thin Gd overlayers" (1989). Peter Dowben Publications. 66.

https://digitalcommons.unl.edu/physicsdowben/66

This Article is brought to you for free and open access by the Research Papers in Physics and Astronomy at DigitalCommons@University of Nebraska - Lincoln. It has been accepted for inclusion in Peter Dowben Publications by an authorized administrator of DigitalCommons@University of Nebraska - Lincoln. 


\title{
Magnetic ordering of thin Gd overlayers
}

\author{
D. LaGraffe and P. A. Dowben* \\ Department of Physics, Syracuse University, Syracuse, New York 13244-1130 \\ M. Onellion* \\ Department of Physics, University of Wisconsin-Madison, Madison, Wisconsin 53706
}

(Received 13 February 1989)

\begin{abstract}
1-6-monolayer-thick films of Gd have been studied with use of synchrotron-radiation photoemission. A method is described that utilizes the linear light polarization to obtain information about the overlayer magnetization. The method is complementary to electron-spin-polarization techniques. The thinner Gd overlayers exhibit a local magnetization component along the surface normal that decreases for thicker overlayers. The Gd films exhibit a $5 d$ exchange splitting that changes from approximately 1.1 to $0.6 \mathrm{eV}$ as the overlayer thickness increases.
\end{abstract}

\section{INTRODUCTION}

Thin metallic films exhibit qualitatively different electronic $^{1,2}$ and magnetic ${ }^{3,4}$ properties compared to their bulk counterparts. One question of widespread interest is the presence, and the details, of magnetic ordering in thin films. Several techniques have been developed by other investigators to study such questions..$^{5-13}$ In this paper we present a method for using linearly polarized synchrotron-radiation photoemission, without electronspin-polarization analysis, to investigate such questions.

Our study was motivated by the realization that there is an unexploited aspect of synchrotron-radiation photoemission that can be used to obtain information regarding the magnetic ordering of overlayers. The method possesses two noteworthy features.

(i) The method is sensitive to any ferrimagnetic ordering, but does not distinguish the type of ferrimagnetism.

(ii) Whether planar or normal magnetization is observed depends on the symmetry character of the particular core and valence orbitals used in the study.

\section{METHODOLOGY}

In order to exploit using linearly polarized synchrotron-radiation photoemission to probe local magnetic ordering, it is essential to first experimentally determine the coupling between the core and valence states involved. This is obtained by using constant-initial-state spectroscopy ${ }^{14}$ (CIS) done at different photon incident angles to select different symmetry states. ${ }^{15}$ The eigenspinors of the relevant orbitals are then calculated to determine the symmetry of the core-level initial states for comparison with the photoemission final states. This can be done analytically for spin-orbit-split levels in the absence of magnetism ordering. ${ }^{16}$ The experimentally determined coupling between the core and valence orbitals then permits us to determine the valence-level spin polarization when combined with the core-level eigenspinors as will be discussed in detail below. Taking CIS spectra across photoemission thresholds is particularly useful for obtaining information about magnetism.

Our procedure begins with calculating the eigenspinors ${ }^{16}$ of the valence- and core-level electrons studied, in our case $\mathrm{Gd} 5 d$ and $\mathrm{Gd} 5 p$ electrons. The calculation yields an analytical solution in the instance of spin-orbit coupling without any magnetic ordering, as other investigators have already established; ${ }^{16}$ the results for the Gd $5 p$ levels are shown in Table I. Four points emerge from examining the eigenspinors.

(1) The occupied Gd $5 d$ level is of mixed spin character.

(2) The Gd $5 p_{3 / 2}, m_{j}=\frac{3}{2}$ or $-\frac{3}{2}$, is of well-defined spin character and contains no $p_{z}$ component, while the Gd $5 p_{1 / 2}$ and the $\mathrm{Gd} 5 p_{3 / 2}, m_{j}=\frac{1}{2}$ or $-\frac{1}{2}$ or $-\frac{1}{2}$, are of mixed spin character and contain a $p_{z}$ component.

(3) The reference axis for these eigenspinors is the spin quantum axis, which is oriented with respect to any spatial axis only when there is magnetic ordering.

TABLE I. The Gd $5 p$ eigenspinors in the presence of a spinorbit interaction and assuming no exchange splitting or magnetization (Ref. 16). $\left(y_{1}^{0}=p_{z}, y_{1}^{1}=p_{x}+i p_{y}, y_{1}^{-1}=p_{x}-i p_{y}\right.$.)

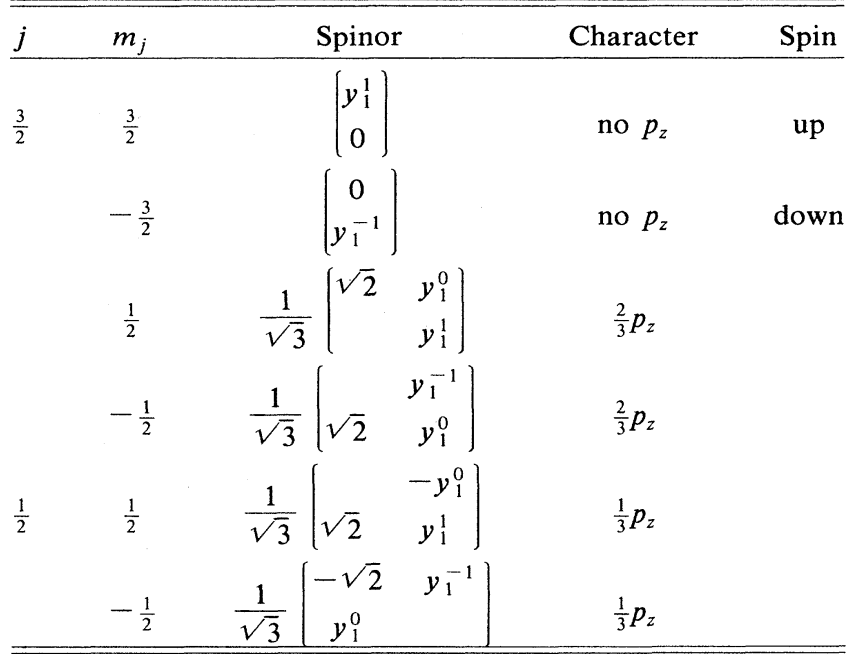

(c) 1989 The American Physical Society 
(4) Both the $5 p_{3 / 2}$ and $5 p_{1 / 2}$ eigenspinors are markedly different compared to the analogous wave functions in the absence (or negligible) of spin-orbit coupling. Such details of the angular momenta coupling have been used by other investigators in studying circularly polarized photoemission and optical spin orientation. ${ }^{17}$

Having obtained the eigenspinors, we performed angle-resolved photoemission experiments and investigated two aspects of the electronic density of states. We have noted how the valence- and core-level features vary in intensity with the incident angle $(\theta)$ between photon vector potential and the surface normal. This provides a direct measure of the symmetry character of the states. This required observing how the valence- and core-level states change their intensity with respect to "reference" features that possess a rough spatial isotropy. In the case of Gd, the $4 f$ multiplet is half-filled and thus all $m_{l}$ levels are filled, providing such a reference feature. We have also studied how the coupling between the valence- and core-level varies with incident angle. In the case of $\mathrm{Gd}$, such a coupling has been established by other investigators $^{18,19}$ as due to an exchange polarization between the Gd $5 d$ and Gd $5 p$ levels. The details of this exchange interaction and the conclusions to be drawn from using different light incidence angles are discussed below.

\section{EXPERIMENT}

We have applied this method to study thin (1-6 monolayer) films of the elemental ferromagnet gadolinium deposited on $\mathrm{Cu}(100)$. The experiments included measuring the crystallographic order by low-energy electron diffraction (LEED), the electronic structure by angleresolved photoemission, and the overlayer cleanliness and possible interdiffusion with angle-resolved Auger-electron spectroscopy. Gd overlayers on $\mathrm{Cu}(100)$ were also deposited on a substrate cooled to $170 \mathrm{~K}$ before deposition. The angle-resolved photoemission measurements were performed using a hemispherical angle-resolved energy analyzer mounted on a two-axis goniometer and possessing a $3^{\circ}$ acceptance full angle. The total energy resolution varied between 0.18 and $0.35 \mathrm{eV}$. Photoemission experiments were performed on a 3-m toroidal-grating monochromator, which is $65 \%$ linearly polarized along the horizontal plane.

1 monolayer of Gd exhibits, in the LEED analysis, diffuse centering spots with four bright spots at the electron momentum of the $\mathrm{Cu}(100)$ first-order spots. Thicker Gd overlayers exhibit only diffuse scattering. These results indicate that one monolayer Gd overlayers possess small domains of $c(2 \times 2)$, misaligned with respect to each other. The thicker Gd films do not exhibit crystallographic order in LEED.

The detailed results of the angle-resolved Auger study are reported elsewhere; ${ }^{20}$ the conclusion is that there is little or no interdiffusion of the $\mathrm{Gd}$ on the $\mathrm{Cu}$ or $\mathrm{Ni}$ substrates, at room temperature or at $170 \mathrm{~K}$. Specifically, there is no observable interdiffusion until the substrate is heated to $60-80^{\circ} \mathrm{C}$; for higher temperatures, however, there is unambiguous interdiffusion. ${ }^{20}$ Auger-electron spectroscopy also indicated less than 1 at. \% contamination (mostly oxygen).

The depositions were made in the absence of an applied magnetic field, to determine whether there is any remnant local magnetic ordering. The ambient magnetic field was measured as approximately $0.05 \mathrm{G}$.

\section{RESULTS}

Figure 1 illustrates photoemission electron energy distribution curves taken at normal emission for three Gd overlayers of increasing thickness. The thickness, in monolayers (labeled ML in figures), is noted in the figure. The spectra exhibit features corresponding to the $\mathrm{Gd} 5 d$ and $6 s$ states $(0.3-0.4 \mathrm{eV}$ binding energy), the $\mathrm{Cu} 3 d$ states $(3.6 \mathrm{eV})$, and the $\mathrm{Gd} 4 f$ states $(8.6 \mathrm{eV})$. All binding energies are referenced to the Fermi energy. As an aside, the feature visible in some of the spectra at approximately $5.6 \mathrm{eV}$ binding energy is intrinsic, not due to contamination, as is discussed in detail elsewhere. ${ }^{21}$

Particularly noteworthy is the enhanced emission from the Gd $5 d$ state, compared to emission from either the $\mathrm{Cu}$ $3 d$ or Gd $4 f$ states, when the light $p$ polarization, $P$, increases from 0.22 to 0.64 , where $P=A /(A+B)$, and $A$ is the amplitude of the photon vector potential along the surface normal ( $p$-polarized light), and $B$ is the amplitude of the photon vector potential in the surface plane $(s-$

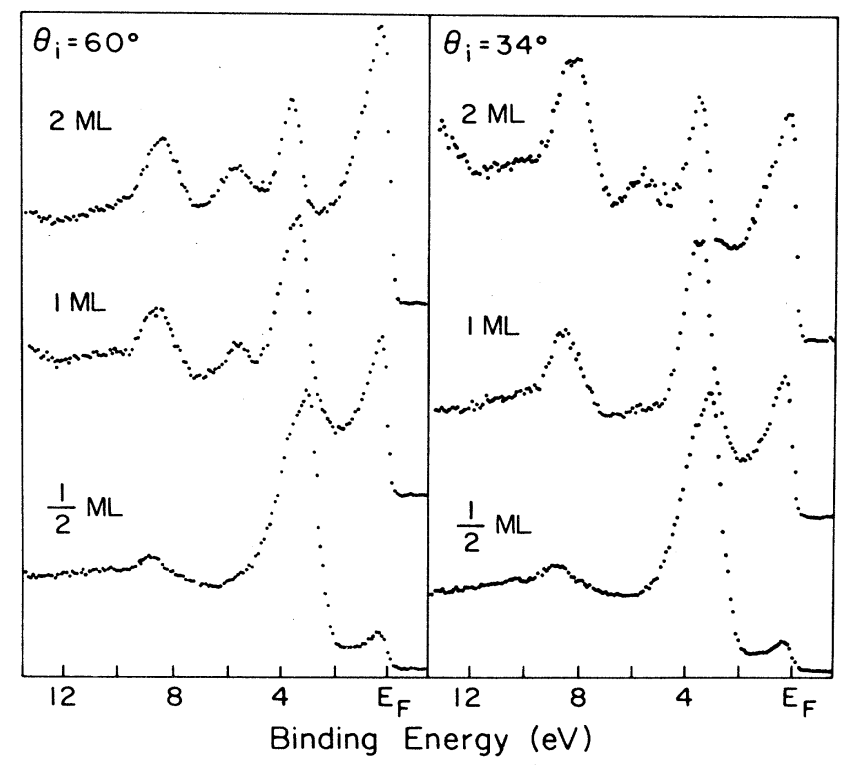

FIG. 1. Energy distribution curves (EDC's) of Gd overlayers on $\mathrm{Cu}(100)$ for photon energy of $33 \mathrm{eV}$ at normal emission. The overlayer thickness, in monolayers (ML), is noted. Both $60^{\circ}$ incident angle (mostly p-polarized light) and $34^{\circ}$ incident angle (mostly s-polarized light) spectra are shown. The spectra exhibit peaks at $0.3-0.4-\mathrm{eV}$ ( $\mathrm{Gd} 5 d$ level), 3.6-eV ( $\mathrm{Cu} 3 d$ level), and $8.0-8.6 \mathrm{eV}$ (Gd $4 f$ level) binding energy. 
polarized light).

For 1-2-monolayer Gd overlayers, the $I(\mathrm{Gd} 5 d): I(\mathrm{Gd}$ $4 f$ ) emission ratio increases $49 \%$ when the light polarization, $P$, is increased from 0.22 to 0.64 . For thicker Gd films, about 6 monolayers, the $I(\mathrm{Gd} 5 d)$ : $I(\mathrm{Gd} 4 f)$ emission ratio increases only $26 \%$, a factor of 2 less. This demonstrates that $p$-polarized light produces an enhancement of emission from the Gd $5 d$ level.

In addition to the $\mathrm{Gd} 5 d$ levels, emission from the $\mathrm{Gd}$ $5 p$ levels was observed to depend on the light polarization. Figure 2 illustrates electron energy distribution curves taken at photon energy $h v=50 \mathrm{eV}$ for two Gd overlayers ( 2 and 5 monolayers), both for the light incident angle $\theta=34^{\circ}(P=0.22$, mostly $s$ polarized $)$ and for $\theta=60^{\circ}(P=0.54$, mostly $p$ polarized $)$. The spectra include the Gd $5 p$ doublet, at binding energies of 21.9 and $26.1 \mathrm{eV}$, and the $\mathrm{Gd} 4 f$ levels, the latter for reference. The spectra exhibit a marked change for the thinner Gd overlayer that does not occur for the thicker Gd overlayer. For the 2-monolayer $\mathrm{Gd}$ overlayer, the higherbinding-energy member of the doublet (the $\mathrm{Gd} 5 p_{1 / 2}$ state) has virtually disappeared for the $\theta=60^{\circ}$ spectrum. For the 5-monolayer Gd overlayer, the $5 p$ doublet is almost independent of $\theta$. (We are using the conventional Russell-Saunders angular momentum notation, although it is not rigorously correct in the present context. ${ }^{22}$ ) The inset illustrates the effect of contamination, and is discussed below.

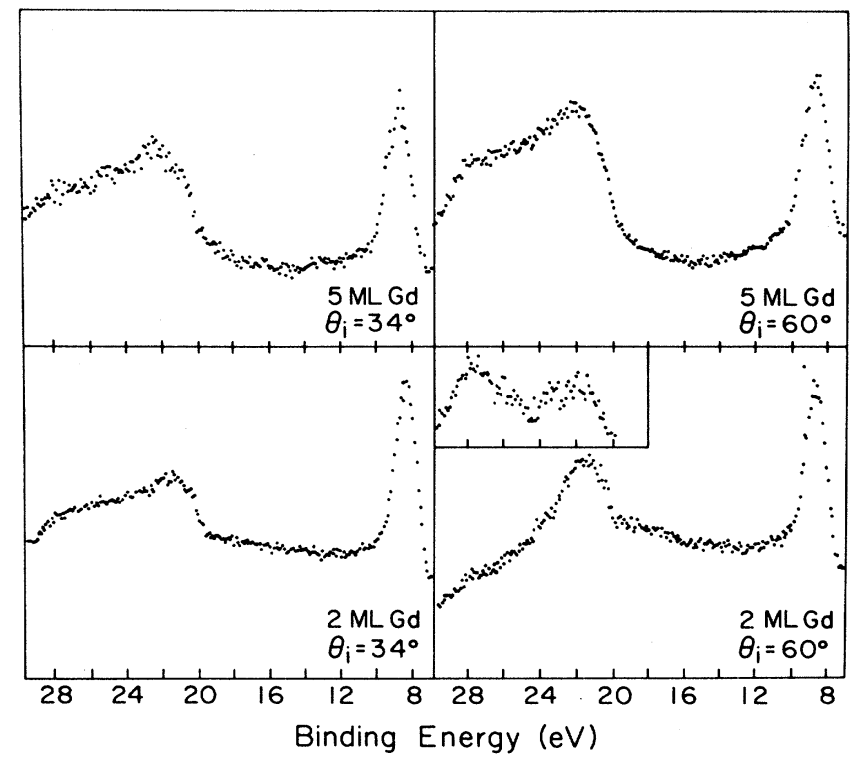

FIG. 2. EDC's of Gd overlayers on $\mathrm{Cu}(100)$ for photon energy of $50 \mathrm{eV}$, taken at normal emission. The overlayer thickness in $\mathrm{ML}$ is noted. Both $60^{\circ}$ and $34^{\circ}$ incident angle results are shown. The spectra exhibit peaks due to the Gd $4 f$ level (approximately $8 \mathrm{eV}$ binding energy) and the Gd $5 p$ spin-orbit doublet between 20 and $28 \mathrm{eV}$. The inset illustrates the 2-ML, $\theta=60^{\circ} 5 p$-level results $3 \mathrm{~h}$ after deposition, long enough for contamination to affect the EDC.
TABLE II. The inflection points for the Gd $5 d$ CIS spectra of Fig. 3 as a function of overlayer thickness in monolayers $(\mathrm{ML})$ and light polarization, across the $5 p_{1 / 2}$ threshold. Also shown are the exchange splittings deduced from these inflection points. See text and references for additional discussion.

\begin{tabular}{cccc}
\hline \hline $\begin{array}{c}\text { Overlayer } \\
\text { thickness } \\
\text { ML }\end{array}$ & \multicolumn{2}{c}{$5 p_{1 / 2}$ inflection } & $\begin{array}{c}\text { Exchange } \\
\text { points }\end{array}$ \\
& $\begin{array}{c}s \text {-pol. } \\
\text { splitting }\end{array}$ & $\begin{array}{c}p \text {-pol. } \\
(\mathrm{eV})\end{array}$ & $(\mathrm{eV})$ \\
\hline 1 & 31.5 & 32.6 & 1.1 \\
2 & 31.5 & 32.4 & 0.9 \\
3 & 31.6 & 32.3 & 0.7 \\
6 & 32.0 & 32.6 & 0.6 \\
\hline
\end{tabular}

Figure 1 illustrates the angular momentum character of the $\mathrm{Gd} 5 d$ state observed by comparing $s$ - and $p$ polarized spectra. A more complete picture of the Gd $5 p$ level is provided in Fig. 3, which illustrates the constant-initial-state ${ }^{14}$ (CIS) spectra of the Gd $5 d$ level from 1-6-monolayer Gd films. Both $\theta=34^{\circ}(P=0.22)$ and $\theta=60^{\circ}(P=0.64)$ spectra are included. A marked change occurs across the $5 p_{1 / 2}$ threshold in comparing 1and 4- monolayer films. For $s$-polarized light, there is an enhancement across both thresholds, consistent with earlier reports by Murgai et al. ${ }^{19}$ who showed electron en-

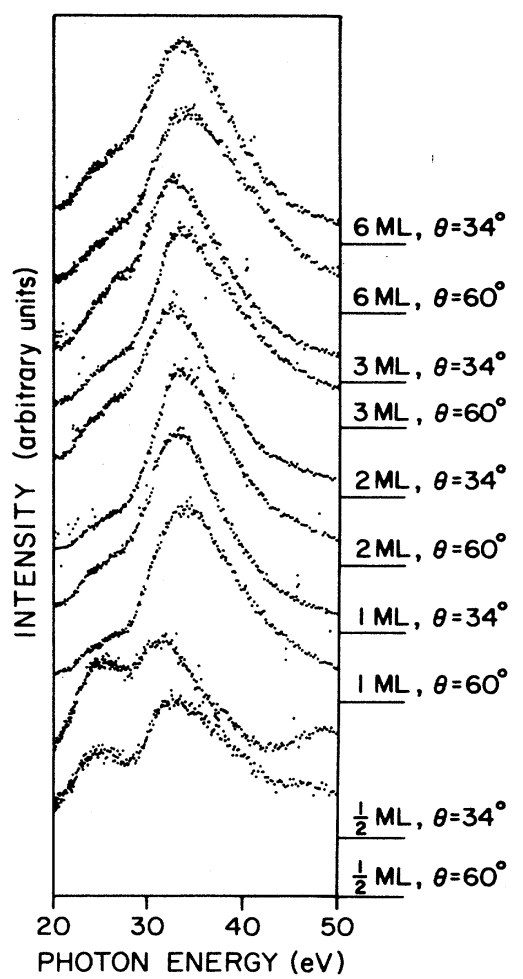

FIG. 3. A series of Gd $5 d$ CIS spectra taken for both $\theta=60^{\circ}$ and $34^{\circ}$. The photon incident angle and the overlayer thickness [in monolayers $(\mathrm{ML})$ ] is noted. All results illustrated were obtained at normal emission. 


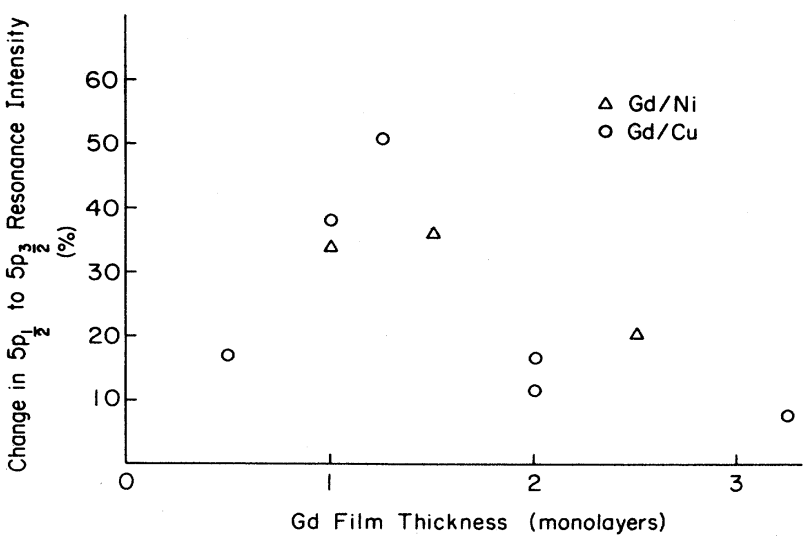

FIG. 4. The fractional change of the Gd $5 d$ emission as a function of overlayer thickness when comparing results obtained using mostly $s$-and $p$-polarized light. See text for formula used to compute the fractional change.

ergy distribution curves. For $p$-polarized light, however, the enhancement across the $5 p_{3 / 2}$ threshold is largely suppressed for 2-monolayer and thinner films.

Figure 3 illustrates that the resonant enhancement of the Gd $5 d$ level across the $5 p_{3 / 2}$ threshold $^{20}$ is largely absent for thin (1-2 monolayer) Gd layers and $p$-polarized light. Further, the $5 p_{1 / 2}$ resonant enhancement of a $\mathrm{Gd}$ overlayer consistently occurs at a higher photon energy for $p$-polarized light than for $s$-polarized light, as summarized in Table II. This result bears on the question of exchange splitting and is discussed below.

Figure 4 displays the fractional change of the resonant $5 d$ emission across the $5 p_{1 / 2}$ and $5 p_{3 / 2}$ thresholds versus overlayer thickness between $s$ - and $p$-polarized light. The fractional change is defined by

$$
F=1.0-a / b \text {, }
$$

where $a$ is the ratio of the $5 d$ resonant emission above the $5 p_{1 / 2}$ versus the $5 p_{3 / 2}$ threshold at $60^{\circ}$ light incident angle and $b$ the $5 d$ ratio of resonant emissions at $34^{\circ}$ incident angle. Since the fractional change is obtained from the raw $5 d$ CIS emission data, without background subtraction, it underestimates the effect of light polarization on the $5 d$ ratios of the resonant emissions. In the figure, a fractional change of 1.0 would mean that the $5 d$ level has no $5 p_{3 / 2}$ resonant emission for $p$-polarized light, while a fractional change of 0.0 would mean that the $5 d$ resonant emission across the $5 p$ thresholds is independent of light polarization. Figure 4 demonstrates that $s$ polarized light enhances emission from the Gd $5 d$ level at the photon energy corresponding to the $5 p_{3 / 2}$ resonant enhancement.

\section{DISCUSSION}

The results illustrated by Fig. 1 bear directly on the symmetry character of the Gd $5 d$ level. Increasing the photon incident angle favors selection of the $d_{3 z^{2}-r^{2}}$ and $p_{z}$ states over other $p$ and $d$ states. ${ }^{15,23}$ These spectra demonstrate that the $\mathrm{Gd} 5 d$ band on $\mathrm{Cu}(100)$ and on $\mathrm{Ni}$ overlayers on $\mathrm{Cu}(100)$ is predominantly of $5 d_{3 z^{2}-r^{2}}$ character.

We have studied the Gd $5 d$ CIS spectra as a function of overlayer thickness. These CIS spectra exhibit a pronounced resonance attributable to excitations from the $5 p$ core levels to the $5 d$ band. ${ }^{20}$ For the 1-2-monolayer Gd films, the $5 p_{3 / 2}$ resonant enhancement is largely suppressed for $p$-polarized light, as noted in Figs. 3 and 4. For the thicker Gd films, however, the $5 p_{3 / 2}$ resonant enhancement is present. Indeed, the CIS spectra for the four-six-monolayer Gd films appear virtually independent of incidence angle.

The changes in the $5 p$ branching ratio for the thin Gd overlayer indicate that the $5 p_{1 / 2}$ feature appears to possess little $p_{z}$ character. Here the branching ratio means the integrated intensity of the $5 p_{3 / 2}$ level divided by that of the $5 p_{1 / 2}$ level, as illustrated in Fig. 2. By contrast, for the thicker (6-monolayer) Gd overlayer, the angular momentum characters of the $5 p_{1 / 2}$ and $5 p_{3 / 2}$ features are quite similar. The inset of Fig. 2, which illustrates spectra taken on a film $3 \mathrm{~h}$ after deposition, reveals the buildup of contamination. This contamination drastically reduces the observed branching ratio, demonstrating that the large branching ratio is not due to contamination. The branching ratio is a consequence of $5 p-5 d$ coupling in the final state. ${ }^{20}$

The results in Figs. 2 and 3 represent a qualitative change in the Gd $5 d-5 p$ coupling between thinner (1-2 monolayer) and thicker (3-6 monolayer) Gd overlayers. The qualitative change in this coupling bears directly on the question of magnetic order.

The analytical solutions for the $5 d$ and $5 p$ eigenspinors (Table I) imply that both features of the $5 p$ doublet should exhibit the same fractional $p_{z}$ character. Further, the solutions imply that the $5 d-5 p$ coupling should be virtually identical when there is no well-defined $5 d$ spin moment, or if the spin angular momentum is randomly oriented in space.

The experimental results (Figs. 2 and 3), however, are quite different. The $5 p_{3 / 2}$ levels exhibit a clear $p_{z}$ character for all $\mathrm{Gd}$ overlayers, while the $5 p_{1 / 2}$ level exhibits virtually no $p_{z}$ character for thinner layers and exhibits similar angular momentum character for the thicker overlayers. This would suggest that the $5 p_{1 / 2}$ level, for thinner films, is predominantly of $p_{x}+i p_{y}$ or $p_{x}-i p_{y}$ character. We conclude that exchange-polarization interaction between $\mathrm{Gd} 5 d$ and $\mathrm{Gd} 5 p$ levels imparts $3 z^{2}-r^{2}$ character to the $5 p_{3 / 2}$ level, which is indistinguishable from $p_{z}$ character in photoemission. ${ }^{18}$

The changes in the Gd $5 d$ CIS spectra with overlayers thickness also demonstrate that there is a qualitative difference in the polarization exchange coupling between the Gd $5 d-5 p_{3 / 2}$ and $5 d-5 p_{1 / 2}$ levels. In particular, the effect of $5 d-5 p_{3 / 2}$ coupling on $5 d$ emission is markedly reduced for $p$-polarized light (which selects $p_{z}$ orbitals, initial states in solid-state terminology) compared to $s$ polarized light (which selects $p_{x}+i p_{y}$ or $p_{x}-i p_{y}$ orbitals, initial states in solid-state terminology). This is observed in spite of the fact that the $5 d$ level itself is clearly (Fig. 1) enhanced by $p$-polarized light. The $5 d$ level itself is 
demonstrably (Fig. 1) of $d_{3 z^{2}-r^{2}}$ symmetry. The assignment of character assumes $C_{4 v}$ point-group symmetry and is applicable only at normal emission.

The conclusion is that the results obtained in Fig. 3 are due to a final-state effect, in which the $5 d$ level couples in a different way to the $5 p$ holes. Examining the $5 p$ eigenspinors (Table I), note that the $5 p_{3 / 2}$ level includes two eigenspinors $\left(m_{j}=+\frac{3}{2}\right.$ and $\left.-\frac{3}{2}\right)$ possessing well-defined spin. These are the only two $5 p$ eigenspinors with welldefined spin. The remaining eigenspinors are remarkably similar, so the differences in the $5 d-5 p$ interaction is likely associated with these two states. The only consistent way to yield such a difference in $5 d-5 p$ coupling is for the $5 d$ level to possess a well-defined spin. With such a spatially well-defined spin, the $5 d$ level can couple effectively to the $5 p_{3 / 2}, m_{j}=\frac{3}{2}$ or $-\frac{3}{2}$, levels. The $5 p_{1 / 2}$ and the $5 p_{3 / 2}$, $m_{j}=\frac{1}{2}$ or $-\frac{1}{2}$ levels, however, do not possess well-defined initial-state spin eigenstates, and the $5 d$ level does not couple equally effectively to them.

This coupling of the rare-earth $5 d$ level with rare-earth core levels is consistent with the earlier work of other investigators, particularly in the studies of the $5 d-5 s$ and $5 d-4 s$ levels. ${ }^{24,25}$ Such coupling also explains the anomalous result that the $5 p_{1 / 2}$ levels exhibit no apparent $p_{z}$ character, whereas (Table I) the analytical eigenspinors yield a definite $p_{z}$ character. The eigenspinors in Table I assume no $5 d-5 p$ coupling and, in particular, no exchange coupling.

The results of Figs. 1-3 are similar to earlier work on $\mathrm{Ni}$ by other investigators, ${ }^{26}$ whose work led us to realize the general utility of polarization-dependent CIS studies. The earlier work ${ }^{26}$ reported that spin polarization of the $\mathrm{Ni} d$ band was enhanced across the $\mathrm{Ni} 3 p$ threshold due to a final-state, exchange-polarization coupling between the Ni $3 d$ electrons and the Ni $3 p$ hole. Ni $3 p$ electrons at the $3 p$ threshold are excited to a minority-spin valence state. In the present case, the $5 p_{3 / 2}$ and $5 p_{1 / 2}$ thresholds just above the Fermi level excite to majority-spin final states, as is discussed in more detail below. This aspect, a threshold to an unoccupied $5 d$ state of well-defined spin, also accounts for the different intensities in the $5 d$ CIS spectra for $s$-polarized and $p$-polarized light observable in Fig. 3. The physics involved is essentially the same as reported by Clauberg et al. ${ }^{26}$ for $\mathrm{Ni}$. In both instances, the $p_{1 / 2}$ level is largely spin unpolarized, and exhibits a larger resonant intensity than the $p_{3 / 2}$ level, which is, in the final state, strongly spin polarized. In the present case, the $p_{3 / 2}, m_{j}=+\frac{3}{2}$ or $-\frac{3}{2}$, levels are of $p_{x}+i p_{y}$ and $p_{x}-i p_{y}$ character and also possess definite spin orientation (Table I). $s$-polarized light selects $p_{x}+i p_{y}$ or $p-i p_{y}$ states, while $p$-polarized light selects $p_{z}$ states. The enhancement of the Gd $5 d$ CIS spectra for $s$-polarized light is due to Fermi's golden rule, which again implies a strong coupling between initial and final states of welldefined spin. ${ }^{18}$

The comparison of spatial anisotropy with the photoemission geometry used to perform our experiments allows us to conclude that the thinner (1-2 monolayer) films exhibit a component of magnetization along the surface normal, while the thicker (4-6 monolayer) films do not. Because of our use of $s$ - and p-polarized light, the method we employed establishes the surface normal as the relevant symmetry axis. If the magnetization vector is zero, randomly oriented in space, or perpendicular to the surface normal, then the $p_{3 / 2}, m_{j}=+\frac{3}{2}$ or $-\frac{3}{2}$, states would not be selected preferentially by $s$-polarized light, in disagreement with our results, which demonstrate such a preferential selection.

Our use of the CIS (Refs. 14 and 15) technique also leads to an estimate of the minimum exchange splitting for $\mathrm{Gd}$ overlayers on $\mathrm{Cu}(100)$. To deduce the exchange splitting, we note that the Gd $5 d$ CIS spectra for $s$ - and p-polarized light, illustrated in Fig. 3, exhibit enhanced resonant intensities at different photon energies. The maxima in the CIS spectra for $s$ - and $p$-polarized light also occur at slightly different photon energies. For consistency of measurement, we used the inflection point between the upward slope and the peak of the $5 p_{1 / 2}$ resonance as a marker. We have chosen the $5 p_{1 / 2}$ resonance to preferentially select for $p_{1 / 2}$ to $d_{3 / 2}$ intra-atomic excitations. $^{20}$ Table II provides the photon energies at which inflection points occur for $s$ - and $p$-polarized light as a function of overlayer thickness in monolayers. Since overall wave-function symmetry must be conserved (as a consequence of Fermi's golden rule), $s$-polarized light produces transitions between $p_{x}+i p_{y}$ to unoccupied $d_{x z}, d_{y z}$ states. $p$-polarized light produces transitions between $p_{z}$ states and unoccupied $d_{3 z^{2}-r^{2}}$ states. Assuming that core levels of different symmetries are close to degenerate ${ }^{27}$ the difference in the CIS peaks is the difference in the unoccupied $d_{x z}, d_{y z}$ and the unoccupied $d_{3 z^{2}-r^{2}}$ levels. By placing the unoccupied $d_{x z}, d_{y z}$ levels just above the Fermi energy, this difference is the minimum estimate of the exchange splitting consistent with our data. The exchange splitting determined in this manner is thickness dependent. As the thickness of the film increases, the measured exchange splitting decreases from 1.1 for 1 monolayer to $0.6 \mathrm{eV}$ for a 6 -monolayer film. This latter value is consistent with the measured value for bulk $\mathrm{Gd}$ of $0.6-0.7 \mathrm{eV} .^{28,29}$

Figure 5 illustrates the energy-level diagram expected for the Gd $5 d$ levels in the presence of an exchange splitting. Earlier calculations, albeit for bulk Gd, imply that the $5 d$ levels, in the absence of an exchange splitting, are virtually degenerate. ${ }^{27}$ In summary, in the presence of an exchange splitting, the relevant $5 d$ energy levels will be ordered as illustrated in Fig. 5. As already discussed, we observed a $5 d_{3 z^{2}-r^{2}}$ state in our spectra, located very near the Fermi energy. To obtain an estimate for the exchange splitting, we use the difference in the Gd $5 d$ CIS between $s$ and $p$-polarized light noted in Table II. The difference just noted is the difference in energy between the $5 d_{x z, y z}$ (majority-spin) and the $5 d_{3 z^{2}-r^{2}}$ (minorityspin) states. We are not certain of the energy location of the $5 d_{x z, y z}$ (spin-majority) state. We used the approximation that the $5 d_{x z, y z}$ (majority-spin) state is almost degenerate with the $5 d_{3 z^{2}-r^{2}}$ (majority-spin) state, the former located immediately above and the latter immediately below the Fermi energy.

An enhanced exchange splitting of ferromagnetic over- 


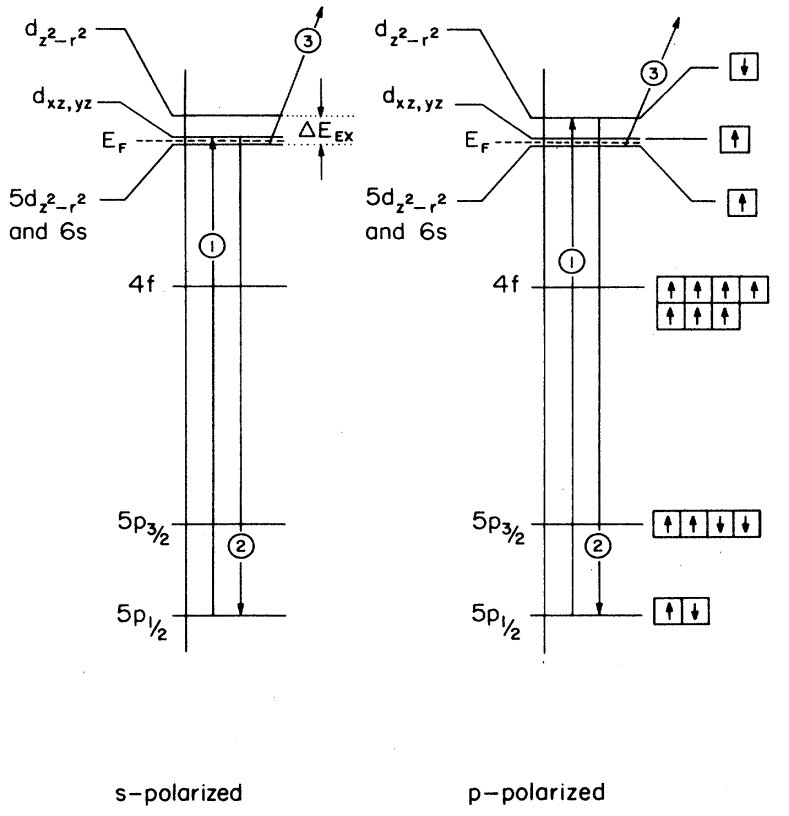

FIG. 5. The Gd energy-level diagram deduced from our results, in the presence of an exchange splitting. The Gd $5 d$ multiplet, the Gd $4 f$ level, and the Gd $5 p$ doublet were included, Also illustrated is the two-step process by which a Gd $5 p$ electron can lead to enhanced emission from the $\mathrm{Gd} 5 d$ level. See text for additional discussion and references.

layers compared to the bulk material has been previously observed in $\mathrm{Ni}$ overlayers on $\mathrm{Cu}(100),{ }^{30}$ and is consistent with earlier calculations on bulk $\mathrm{Gd}^{31}$ and the enhancement expected for thin films. ${ }^{2}$ It is noteworthy that the exchange constant that we have deduced from our results decreases with overlayer thickness, as does the surfacenormal magnetization component. Gd overlayers on $\mathrm{Ni} / \mathrm{Cu}(100)$ also exhibit an exchange splitting, for which work is in progress.

Excitations from the Gd $5 p$ levels to the Gd $6 s p$ band are not an important contribution to the interband transitions and the resonance photoemission results discussed above. This is in part a consequence of the high electron occupancy of the Gd $6 s p$ band, and the large bandwidth (more than $3 \mathrm{eV}$ ). Symmetry restrictions in s-polarizedlight incidence geometry do not allow for any excitations to this band. ${ }^{18,15}$ Other researchers have also shown that transitions to the Gd $6 s p$ band are very weak. ${ }^{29}$ Transition probabilities based upon dipole selection rules strongly favor $p_{1 / 2}$-to- $d_{3 / 2}$ transitions over $p_{1 / 2}$-to-s $s_{1 / 2}$ transitions.

The change in resonance enhancement is directly related to the (local) surface-normal component of the magnetization vector as illustrated in Fig. 4. The surfacenormal magnetization component is largest for a 1monolayer film. As the overlayer thickness grows, and the disorder increases, the surface-normal magnetization decreases. For submonolayer films the surface-normal magnetization component also decreases, consistent with imperfectly arranged islands. The results obtained are similar for $\mathrm{Gd}$ overlayers on $\mathrm{Cu}(100)$ and $\mathrm{Ni}$ overlayers on $\mathrm{Cu}(100)$.

It is worth emphasizing the limitations of the results discussed above. The present investigation utilizes linearly polarized synchrotron-radiation photoemission. This combination of left-handed and right-handed circularly polarized light limits the information that can be obtained. ${ }^{17}$ Specifically, the present approach allows us to determine the presence of a well-defined local surfacenormalization magnetization component. It does not provide information concerning the spatial extent of the magnetic ordering. Further, the approach does not provide information concerning the precise type of ferrimagnetic ordering. The method presented here is only sensitive to the presence of (local) magnetic ordering, without specifying the nature of the long-range magnetic order. Further, since we are changing the vector potential with respect to the surface normal in our measurements, we are defining the surface normal as the symmetry axis. The approach is, therefore, sensitive to the presence of a magnetization vector component along the surface normal. Finally, our method provides an estimate of the exchange splitting. Our method is complementary to the techniques other investigators ${ }^{32-35}$ have used to study the magnetic properties of Gd surfaces and overlayers.

In summary, we have used polarization-dependent photoemission to obtain results for the presence of (local) surface-normal magnetization in thin metal overlayers. We have also used the technique of constant-initial-state spectroscopy ${ }^{14,15}$ to deduce an exchange interaction of approximately $1.1 \mathrm{eV}$ for a 1-monolayer film that decreases to $0.6 \mathrm{eV}$ for thicker films. In devising this method, we benefited from the earlier work of several investigators. ${ }^{19,24-26,36}$ The method presented does not distinguish the various ferrimagnetic orientations possible; on the other hand, it is sensitive to any such magnetic orientation. In this respect, the method is complementary to electron-spin-polarized techniques, ${ }^{5-13,24,25,27,28,31,32}$ which provide more detailed information for ferromagnetic systems, but are not sensitive to other ferrimagnetic orientations, in particular, antiferromagnetism. The essential elements of the method require well-known valence and core-level eigenspinors, and symmetry characters of such states that permit the use of polarization-dependent photoemission to infer the presence of magnetic orientation. At present, we are not certain what spatial range of magnetic ordering is observable with this method. What has been demonstrated is that other investigators can use this method for studying the presence or absence of a local surface-normal magnetization component for various ferrimagnetic orientations, and for obtaining an estimate of the exchange splitting. As regards the magnetization of the Gd overlayers, the magnetization is consistent with earlier reports from other investigators of $\mathrm{Gd}$ on different substrates that state there is no surface-normal magnetization component for thick films. ${ }^{37}$ The exchange splitting deduced is consistent with the enhancement expected for thin films $s^{2,30,31}$ and the known bulk exchange-splitting value for $\mathrm{Gd}^{27-29}$ 


\section{ACKNOWLEDGEMENTS}

We have benefited from conversations with several researchers, including Peter Flynn, Jeff Hanson, Kristal Hathaway, David Walieczska, John Weaver, and especially Clifford Olson. Financial support was provided by the U.S. Department of Energy and by the Office of $\mathrm{Na}$ - val Research, U.S. Department of Defense. The photoemission experiments were performed at the University of Wisconsin-Madison Synchrotron Radiation Center (Stoughton, WI) (SRC), a National Science Foundation (NSF)-supported facility. We wish to thank the SRC staff, particularly the operations staff and E. Rowe, for their support.
*Authors to whom further correspondence should be addressed.

${ }^{1}$ D. Wang, A. J. Freeman, and H. Krakauer, Phys. Rev. B 24, 1126 (1981); J. Tersoff and L. M. Falicov, ibid. B 26, 6186 (1982); H. Huang, X. Zhu, and J. Hermanson, ibid. B 29, 2270 (1984).

${ }^{2}$ C. L. Fu, A. J. Freeman, and T. Oguchi, Phys. Rev. Lett. 54, 2700 (1985); for a recent review and extensive references, see P. A. Dowben, M. Onellion, and Y. J. Kime, Scanning Electron Microsc. 2, 177 (1988).

${ }^{3}$ Magnetic Properties of Low-Dimensional Systems, edited by L. M. Falicov and J. L. Moran-Lopez (Springer-Verlag, Berlin, 1986).

${ }^{4}$ J. Mathon, Rep. Prog. Phys. 51, 1 (1988).

${ }^{5}$ Polarized Electrons in Surface Physics, edited by R. Feder (World Scientific, Singapore, 1985).

${ }^{6}$ E. Kisker, K. Schroder, M. Campagna, and W. Gudat, Phys. Rev. Lett. 52, 2285 (1984).

${ }^{7}$ D. L. Abraham and H. Hopster, Phys. Rev. Lett. 58, 1352 (1987).

${ }^{8}$ D. L. Abraham and H. Hopster, Phys. Rev. Lett. 59, 2333 (1987).

${ }^{9}$ R. Raue, H. Hopster, and R. Clauberg, Phys. Rev. Lett. 50, 1623 (1983).

${ }^{10}$ D. R. Penn, S. P. Apell, and S. M. Girvin, Phys. Rev. B 32, 7753 (1985).

${ }^{11}$ M. Landolt, R. Allenspach, and M. Tabotelli, Surf. Sci. 178, 311 (1986).

${ }^{12}$ For the magneto-optical Kerr effect, see, e.g., T. Katayama, Y. Suzuki, H. Awano, Y. Nishihara, and N. Koshizuka, Phys. Rev. Lett. 60, 1426 (1988); H. Feil and C. Haas, 58, 65 (1987); S. D. Bader, E. R. Moog, and P. Grunberg, J. Magn. Magn. Mater. 53, L295 (1986).

${ }^{13}$ For neutron scattering, see, e.g., G. P. Felcher, Phys. Rev. B 24, 1595 (1981); J. A. C. Bland, D. Pescia, and R. F. Willis, Phys. Rev. Lett. 58, 1244 (1987).

${ }^{14}$ G. J. Lapeyre, A. D. Baer, J. Hermanson, J. Anderson, J. A. Knapp, and P. L. Gobby, Solid State Commun. 15, 1601 (1974); G. J. Lapeyre, J. Anderson, P. L. Gobby, and J. A. Knapp, Phys. Rev. 33, 1290 (1977).

${ }^{15}$ M. Scheffler, K. Kambe, and F. Forstmann, Solid State Commun. 25, 93 (1978); G. J. Lapeyre and J. Anderson, Surf. Sci. 89, 304 (1979); J. Hermanson, Solid State Commun. 22, 9 (1977); G. Borstel, M. Neumann, and M. Wohlecke, Phys. Rev. B 23, 3121 (1981)

${ }^{16}$ E. Merzbacher, Quantum Mechanics (Wiley, New York,
1970), pp. 389-394.

${ }^{17}$ Optical Orientation, Vol. 8 of Modern Problems in Condensed Matter Sciences, edited by F. Meier and B. P. Zakharchenya (Elsevier, Amsterdam, 1984).

${ }^{18}$ P. A. Dowben, D. LaGraffe, and M. Onellion, J. Phys. C (to be published).

${ }^{19}$ V. Murgai, Y.-S. Huang, M. L. denBoer, and S. Horn, Solid State Commun. 66, 329 (1988).

${ }^{20}$ D. LaGraffe, P. A. Dowben, and M. Onellion, Phys. Rev. B (to be published).

${ }^{21}$ D. LaGraffe, P. A. Dowben, and M. Onellion (unpublished).

${ }^{22}$ Due to the large atomic number $(Z=64)$ of gadolinium.

${ }^{23}$ M. Tinkham, Group Theory and Quantum Mechanics (McGraw-Hill, New York, 1964).

${ }^{24}$ F. R. McFeely, S. P. Kowalczyk, L. Ley, and D. A. Shirley, Phys. Lett. 45A, 227 (1973).

${ }^{25}$ D. A. Shirley, in Photoemission in Solids I, Vol. 26 of Topics in Applied Physics, edited by M. Cardona and L. Ley (SpringerVerlag, New York, 1978), pp. 165ff.

${ }^{26}$ R. Clauberg, W. Gudat, E. Kisker, E. Kuhlmann, and G. M. Rothberg, Phys. Rev. Lett. 47, 1314 (1981).

${ }^{27}$ B. N. Harmon and A. J. Freeman, Phys. Rev. B 10, 1979 (1974).

${ }^{28}$ A. B. Besnosov, V. V. Eremendo, and V. P. Gnezdilov, J. Magn. Magn. Mater. 43, 243 (1984); see also Ref. 36.

${ }^{29}$ G. Schutz, M. Knulle, R. Wienke, W. Wilhelm, W. Wagner, P. Kienle, and R. Frahm, Z. Phys. B 73, 67 (1988).

${ }^{30}$ M. A. Thompson and J. L. Erskine, Phys. Rev. B 31, 6832 (1985).

${ }^{31}$ R. E. Watson, A. J. Freeman, and J. P. Dimmock, Phys. Rev. 167, 497 (1968).

${ }^{32}$ D. Weller, S. F. Alvarado, W. Gudat, K. Schroder, and M. Campagna, Phys. Rev. Lett. 54, 1555 (1985).

${ }^{33}$ M. Taborelli, R. Allenspach, G. Boffa, and M. Landolt, Phys. Rev. Lett. 56, 2869 (1986)

${ }^{34}$ C. Rau and M. Robert, Phys. Rev. Lett. 58, 2714 (1987), and references therein.

${ }^{35}$ C. Carbone and E. Kisker, Phys. Rev. B 36, 1280 (1987).

${ }^{36}$ G. Schonhense, B. Kessler, N. Muller, B. Schmiedeskamp, and U. Heinzmann, Phys. Scr. 35, 541 (1987).

${ }^{37}$ S. H. Liu, in Handbook on the Physics and Chemistry of Rare Earths, edited by K. A. Gschneider, Jr. and L. Eyring (North-Holland, Amsterdam, 1978), Vol. 1, pp. 284ff.; J. L. Erskine and E. A. Stern, Phys. Rev. B 8, 1239 (1973). 